TRANSACTIONS OF THE

AMERICAN MATHEMATICAL SOCIETY

Volume 358, Number 9, September 2006, Pages 3807-3820

S 0002-9947(06)04004-9

Article electronically published on March 24, 2006

\title{
COMPLETE NONORIENTABLE MINIMAL SURFACES IN A BALL OF $\mathbb{R}^{3}$
}

\author{
F. J. LÓPEZ, FRANCISCO MARTIN, AND SANTIAGO MORALES
}

\begin{abstract}
The existence of complete minimal surfaces in a ball was proved by N. Nadirashvili in 1996. However, the construction of such surfaces with nontrivial topology remained open. In 2002, the authors showed examples of complete orientable minimal surfaces with arbitrary genus and one end. In this paper we construct complete bounded nonorientable minimal surfaces in $\mathbb{R}^{3}$ with arbitrary finite topology. The method we present here can also be used to construct orientable complete minimal surfaces with arbitrary genus and number of ends.
\end{abstract}

\section{INTRODUCTION}

This work is part of a series of papers about the admissible topological type of a complete bounded minimal surface in $\mathbb{R}^{3}$. The first example of such a surface, due to Nadirashvili [5], had the topology of a disk. His proof consisted of successive modifications of the Weierstrass representation of a minimal surface that involved the use of the classic Runge's theorem in the plane. Since Nadirashvili's result, examples with more complicated topological type were constructed: annuli in [4, and orientable surfaces with finite genus and one end in [2]. The second family of examples was obtained by combining Nadirashvili's ideas with a technique that solves period problems for minimal surfaces with arbitrary genus. In this paper we extend this kind of result to the nonorientable case. Actually, we have proved:

Theorem. For any genus $\sigma \geq 1$ and any $k \in \mathbb{N}$, there exists a complete bounded nonorientable minimal surface in $\mathbb{R}^{3}$ with genus $\sigma$ and $k$ ends.

Similar techniques to those developed in [5] and 2] have been applied to the orientable double covering of our nonorientable surfaces. However, in this case the Weierstrass have to satisfy a compatibility condition with the reversing orientable involution. This fact introduces additional difficulties in the treatment of the period problem. To overcome these obstacles, we have had to use, among other things, a general version of Runge's theorem on Riemann surfaces 8].

We would like to point out that the present method can also be used to construct orientable complete minimal surfaces with arbitrary genus and number of ends.

Received by the editors May 24, 2004.

2000 Mathematics Subject Classification. Primary 53A10; Secondary 49Q05, 49Q10, 53 C42.

Key words and phrases. Complete bounded minimal surfaces, nonorientable minimal immersions.

This research was partially supported by MEC-FEDER Grant No. MTM2004-00160.

(C)2006 American Mathematical Society Reverts to public domain 28 years from publication 3807 


\section{BACKGROUND AND NOTATION}

In this section, we provide some definition and notation that we will use throughout the paper.

2.1. Metrics and divisors on a Riemann surface. Let $\mathcal{N}$ and $d \hat{s}^{2}$ be a Riemann surface and a Riemannian conformal metric on $\mathcal{N}$, respectively. Given a curve $\alpha$ in $\mathcal{N}$, by length $(\alpha, d \hat{s})$ we mean the length of $\alpha$ with the metric $d \hat{s}^{2}$. Given a subset $W \subset \mathcal{N}$, we define:

- $\operatorname{dist}_{(d \hat{s}, W)}(p, q)=\inf \{\operatorname{length}(\alpha, d \hat{s}) \mid \alpha:[0,1] \rightarrow W, \alpha(0)=p, \alpha(1)=q\}$, for any $p, q \in W$,

- $\operatorname{dist}_{(d \hat{s}, W)}\left(T_{1}, T_{2}\right)=\inf \left\{\operatorname{dist}_{(d \hat{s}, W)}(p, q): p \in T_{1}, q \in T_{2}\right\}$, for any $T_{1}, T_{2} \subset$ $W$,

- $\operatorname{diam}_{d \hat{s}}(W)=\sup \left\{\operatorname{dist}_{(d \hat{s}, W)}(p, q): p, q \in W\right\}$.

The concepts of the (multiplicative) divisor on $\mathcal{N}$, the integral divisor on $\mathcal{N}$, and the natural partial ordering, $\geq$, on divisors can be found in 1]. Let $\omega$ be a meromorphic function or 1-form on $\mathcal{N}$. Let $W \subset \mathcal{N}$ and suppose that $\omega$ has a finite number of zeroes, $z_{1}, \ldots, z_{n}$, and a finite number of poles, $p_{1}, \ldots, p_{m}$, in $W$. We denote by $\left(\omega_{\left.\right|_{W}}\right)_{0}=z_{1} \cdots z_{n},\left(\omega_{\left.\right|_{W}}\right)_{\infty}=p_{1} \cdots p_{m}$, and $\left(\omega_{\left.\right|_{W}}\right)=\left(\omega_{\left.\right|_{W}}\right)_{0} /\left(\omega_{\left.\right|_{W}}\right)_{\infty}$, the zero divisor, the polar divisor, and the divisor of $\omega$ on $W$, respectively. When $W=\mathcal{N}$, we simply write $(\omega),(\omega)_{0}$, and $(\omega)_{\infty}$, respectively.

2.2. The conformal structure. In this subsection, we describe the family of Riemann surfaces that we will deal with in the next sections.

Let $\sigma \geq 0$ be an integer number, let $d_{1}, \ldots, d_{\sigma+1}$ be a sequence of pairwise distinct complex numbers satisfying $\operatorname{Im} d_{i} \neq 0, i=1, \ldots, \sigma+1$, and let $\bar{M}$ be the algebraic hyperelliptic curve of genus $\sigma$ given by

$$
\bar{M}=\left\{(z, w) \in \overline{\mathbb{C}}^{2}: w^{2}=\prod_{i=1}^{\sigma+1}\left(z-d_{i}\right)\left(z-\overline{d_{i}}\right)\right\} .
$$

Let $I: \bar{M} \rightarrow \bar{M}$ be the antiholomorphic involution without fixed points on $\bar{M}$, given by $I(z, w)=(\bar{z},-\bar{w})$, and label $A(z, w)=(z,-w)$ the hyperellyptic involution.

In what follows $\Delta$ will be an open bounded simply-connected domain in $\mathbb{C}$ whose boundary is a Jordan curve, and such that $\left\{d_{1}, \ldots, d_{\sigma+1}\right\} \subset \Delta$. Observe that $z^{-1}(\overline{\mathbb{C}} \backslash \bar{\Delta})=D_{+} \cup D_{-}$, where $D_{ \pm}$is a conformal disk around $\infty^{ \pm}=(\infty, \pm \infty)$, and $z_{\mid D_{ \pm}}$is one to one. We denote $f_{ \pm}=z_{\mid D_{ \pm}}^{-1}$. Let $\left\{\beta_{1}, \ldots, \beta_{k-1}\right\} \subset \mathbb{C} \backslash \bar{\Delta}$ be a set of real numbers and label $\beta_{i}^{ \pm}=f_{ \pm}\left(\beta_{i}\right), i=1, \ldots, k-1$. We denote $M=\bar{M}-\left\{\beta_{1}^{ \pm}, \ldots, \beta_{k-1}^{ \pm}, \infty^{ \pm}\right\}$.

Now, we are going to describe the domains of $M$ that we will deal with. Given $\alpha$ as a Jordan curve in $\mathbb{C}$, we denote Int $\alpha$ as the bounded connected component of $\mathbb{C} \backslash \alpha$.

Definition 1. By a multigon, we mean a finite family of simple closed polygonal curves in $\mathbb{C}, \mathfrak{P}=\left\{P_{1}, \ldots, P_{k}\right\}$, pairwise disjoint, such that:

- $\bar{\Delta} \subset \operatorname{Int} P_{k}$,

- $\overline{\operatorname{Int} P_{i}} \subset \operatorname{Int} P_{k} \backslash \bar{\Delta}, i=1, \ldots, k-1$,

- $\beta_{i} \in \operatorname{Int} P_{i}, i=1, \ldots, k-1$,

- $\overline{\operatorname{Int} P_{1}}, \ldots, \overline{\operatorname{Int} P_{k-1}}$ are pairwise disjoint. 
We denote Int $\mathfrak{P}$ as the domain of $\mathbb{C}$ whose boundary consists of the curves in $\mathfrak{P}$. See Figure 1

If $\mathfrak{P}$ is a multigon, we write $D(\mathfrak{P}) \subset M$ as the domain whose boundary coincides with the union of the Jordan curves $f_{+}(\mathfrak{P})$ and $I\left(f_{+}(\mathfrak{P})\right)$.

Given $\xi>0$, small enough, we define a new multigon $\mathfrak{P}^{\xi}=\left\{P_{1}^{\xi}, \ldots, P_{k}^{\xi}\right\}$, where $P_{i}^{\xi}, i=1, \ldots, k$, are the parallel polygonal curves to $P_{i}$, satisfying that the distance between parallel sides is equal to $\xi$, and $\overline{\operatorname{Int} \mathfrak{P}^{\xi}} \subset \operatorname{Int} \mathfrak{P}$. Whenever we write $\mathfrak{P}^{\xi}$ in the paper we are assuming that $\xi$ is small enough to $\mathfrak{P}^{\xi}$ were a multigon. Observe that $\overline{D\left(\mathfrak{P}^{\xi}\right)} \subset D(\mathfrak{P})$.

\subsection{The Weierstrass representation of a nonorientable minimal surface.} Finally, we introduce the Weierstrass representation of nonorientable minimal surfaces.

Given $D \subset \bar{M}$ a domain, we will say that a function, or a 1-form, is harmonic, holomorphic, meromorphic, ... on $\bar{D}$, if it is harmonic, holomorphic, meromorphic, ... on a domain containing $\bar{D}$.

Let $D \subset M$ be a domain invariant under $I$, and let $\Phi=\left(\Phi_{1}, \Phi_{2}, \Phi_{3}\right)$ be the Weierstrass representation of a minimal immersion $X: \bar{D} \rightarrow \mathbb{R}^{3}$ such that $I^{*} \Phi=\bar{\Phi}$. As $X \circ I=X$, then it induces a minimal immersion $\mathcal{X}: \overline{\mathcal{D}} \rightarrow \mathbb{R}^{3}$, where $\overline{\mathcal{D}}=\bar{D} /\langle I\rangle$ is a nonorientable surface with boundary. Obviously $X=\mathcal{X} \circ \pi$, where $\pi: \bar{D} \rightarrow \overline{\mathcal{D}}$ is the canonical projection.

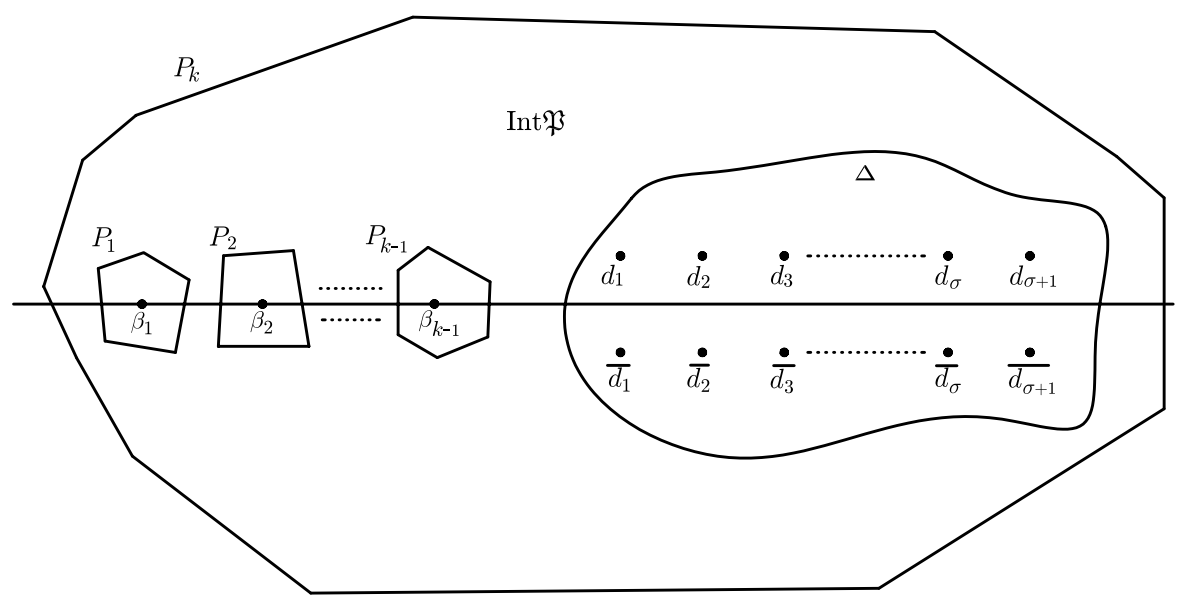

Figure 1. The multigon $\mathfrak{P}$ and the set $\Delta$.

We can write $\Phi_{j}=\varphi_{j}(z, w) \frac{d z}{w}$, where $\varphi_{j}$ is a holomorphic function on $\bar{D}, j=$ $1,2,3$, and denote $\varphi \stackrel{\text { def }}{=}\left(\varphi_{1}, \varphi_{2}, \varphi_{3}\right)$. Note that $\varphi \circ I=-\bar{\varphi}$. With this notation, if we write the Riemannian metric induced by $X$ as $d s_{X}^{2}=\lambda_{X}^{2}\left\|\frac{d z}{w}\right\|^{2}$, then

$$
\lambda_{X}=\frac{1}{\sqrt{2}}\|\varphi\|=\frac{1}{\sqrt{2}} \sqrt{\left|\varphi_{1}\right|^{2}+\left|\varphi_{2}\right|^{2}+\left|\varphi_{3}\right|^{2}}>0 \text { on } \bar{D} .
$$

For the sake of simplicity, given $W \subset M, p, q \in W$ and $T \subset W$, we write $\operatorname{dist}_{(X, W)}(p, q)$ and $\operatorname{dist}_{(X, W)}(p, T)$ instead of $\operatorname{dist}_{\left(d s_{X}, W\right)}(p, q)$ and $\operatorname{dist}_{\left(d s_{X}, W\right)}(p, T)$, respectively. 


\section{Previous lemmas}

Using the previous notations, a precise statement of our main result is the following.

Theorem 1. For any $\sigma \geq 0$ and $k \geq 1$, there exists a complete bounded minimal immersion $\mathcal{X}: \mathcal{D} \rightarrow \mathbb{R}^{3}$, where $\bar{D} \subset M, I(D)=D, \mathcal{D}=D /\langle I\rangle$ and $\mathcal{D}$ is homeomorphic to a nonorientable compact surface of genus $\sigma+1$ minus $k$ points.

In order to get this theorem, we need the following two lemmas.

Lemma 1. Consider a multigon $\mathfrak{P}$ and a minimal immersion $F: \overline{D(\mathfrak{P})} \rightarrow \mathbb{R}^{3}$ whose Weierstrass representation $\Phi$ satisfies $I^{*}(\Phi)=\bar{\Phi}$. Let $K_{1}, K_{2}$ be compact subsets in $D(\mathfrak{P})$ satisfying the facts that $K_{1}, K_{2}$ and $I\left(K_{1}\right)$ are pairwise disjoint, $I\left(K_{2}\right)=K_{2}, D\left(\mathfrak{P}^{\vartheta}\right) \subset K_{2}$, for some $\vartheta>0$, and the set $\left\{\beta_{1}^{ \pm}, \ldots, \beta_{k-1}^{ \pm}, \infty^{ \pm}\right\}$has a (unique) point in each connected component of $\bar{M} \backslash\left(K_{1} \cup K_{2} \cup I\left(K_{1}\right)\right)$.

Then for any $\alpha>0$ there exists $h: \overline{D(\mathfrak{P})} \rightarrow \mathbb{C}$, a holomorphic function without zeroes, such that:

1. $h \circ I=1 / \bar{h}$,

2. $|h-\alpha|<1 / \alpha$ in $K_{1}$,

3. $|h-1|<1 / \alpha$ in $K_{2}$,

4. the Weierstrass data $\widetilde{\Phi}$ given by $\widetilde{g}=g / h$ and $\widetilde{\Phi}_{3}=\Phi_{3}$ satisfy $I^{*}(\widetilde{\Phi})=\widetilde{\widetilde{\Phi}}$, and the associated minimal immersion $\widetilde{F}: \overline{D(\mathfrak{P})} \rightarrow \mathbb{R}^{3}$ is well defined.

Lemma 2. Let $\mathfrak{P}$ be a multigon and let $r>0$. Consider a minimal immersion $X: \overline{D(\mathfrak{P})} \rightarrow \mathbb{R}^{3}$ satisfying:

1. $X=\operatorname{Re}\left(\int_{p_{0}} \Phi\right)$, where $p_{0}=\left(d_{1}, 0\right.$ and $I^{*} \Phi=\bar{\Phi}$;

2. $\|X\|<r$ in $\overline{D(\mathfrak{P})}$.

Then, for any $\varepsilon, s>0$ such that $\mathfrak{P}^{\varepsilon}$ is a multigon, there exist a multigon $\tilde{\mathfrak{P}}$ and a conformal minimal immersion $Y: \overline{D(\widetilde{\mathfrak{P}})} \rightarrow \mathbb{R}^{3}$, such that:

1. $\overline{D\left(\mathfrak{P}^{\varepsilon}\right)} \subset D(\widetilde{\mathfrak{P}}) \subset \overline{D(\widetilde{\mathfrak{P}})} \subset D(\mathfrak{P})$,

2. $Y=\operatorname{Re}\left(\int_{p_{0}} \widetilde{\Phi}\right)$, where $\widetilde{\Phi}$ satisfies $I^{*}(\widetilde{\Phi})=\overline{\widetilde{\Phi}}$,

3. $\operatorname{dist}_{(Y, \overline{D(\widetilde{\mathfrak{P}})})}\left(\partial(D(\widetilde{\mathfrak{P}})), \partial\left(D\left(\mathfrak{P}^{\varepsilon}\right)\right)\right)>s$,

4. $Y(D(\widetilde{\mathfrak{P}})) \subset B_{R}, \quad R=\sqrt{r^{2}+(2 s)^{2}}+\varepsilon$,

5. $\|Y-X\|<\varepsilon$ in $D\left(\overline{\mathfrak{P}^{\varepsilon}}\right)$.

3.1. Proof of Lemma 1. First, we construct a basis $\mathcal{B}=\left\{\gamma_{1}, \ldots, \gamma_{\sigma+k}, \Gamma_{1}\right.$, $\left.\ldots, \Gamma_{\sigma+k-1}\right\}$ of $H_{1}(D(\mathfrak{P}), \mathbb{R})$ contained in $K_{2}$, and satisfying $I_{*}\left(\gamma_{j}\right)=\gamma_{j}$ and $I_{*}\left(\Gamma_{j}\right)=-\Gamma_{j}$.

To do this, consider Jordan curves $\hat{c}_{1}, \ldots, \hat{c}_{\sigma}, c_{1}, \ldots, c_{\sigma+k}$ in the $z$-plane satisfying:

- The winding number of $\hat{c}_{j}$ around $d_{j}$ and $d_{j+1}$ is 1 , and the winding number around $\beta_{l}, \overline{d_{h}}$ and $d_{i}$ is $0, l=1, \ldots, k-1, h=1, \ldots, \sigma+1$, and $i=$ $1, \ldots, j-1, j+2, \ldots, \sigma+1$.

- If $j \leq \sigma$ the winding number of $c_{j}$ around $d_{i}$ and $\overline{d_{i}}$ is $\delta_{i, j}, i=1, \ldots, \sigma+1$, and the winding number around $\beta_{l}$ is $0, l=1, \ldots k-1$. 
- If $\sigma<j<\sigma+k$ the winding number of $c_{j}$ around $\beta_{i}$ is $\delta_{i, j-k}, i=1, \ldots, k-1$, and the winding number around $d_{l}, \overline{d_{l}}$ is $0, l=1, \ldots \sigma+1$.

- The winding number of $c_{\sigma+k}$ around $\beta_{i}, d_{j}, \overline{d_{j}}$ is $-1, i=1, \ldots, k-1$, $j=1, \ldots, \sigma+1$.

Take $\hat{\alpha}_{j}$ and $\alpha_{j}$ as curves in $K_{2}$ obtained by lifting $\hat{c}_{j}$ and $c_{j}$, respectively, for any $j$. Then, we define $\gamma_{j} \stackrel{\text { def }}{=} \frac{1}{2}\left(\alpha_{j}+I_{*}\left(\alpha_{j}\right)\right)$, for $j=1, \ldots, \sigma+k$, and

$$
\Gamma_{j}= \begin{cases}\frac{1}{2}\left(\hat{\alpha}_{j}-I_{*}\left(\hat{\alpha}_{j}\right)\right), & j=1, \ldots, \sigma, \\ \frac{1}{2}\left(\alpha_{j}-I_{*}\left(\alpha_{j}\right)\right), & j=\sigma+1, \ldots, \sigma+k-1 .\end{cases}
$$

Note that $\gamma_{j}=\alpha_{j}, j=1, \ldots, \sigma$. It is not hard to check that $\mathcal{B}$ is indeed a basis of $H_{1}(D(\mathfrak{P}), \mathbb{R})$, satisfying the required conditions.

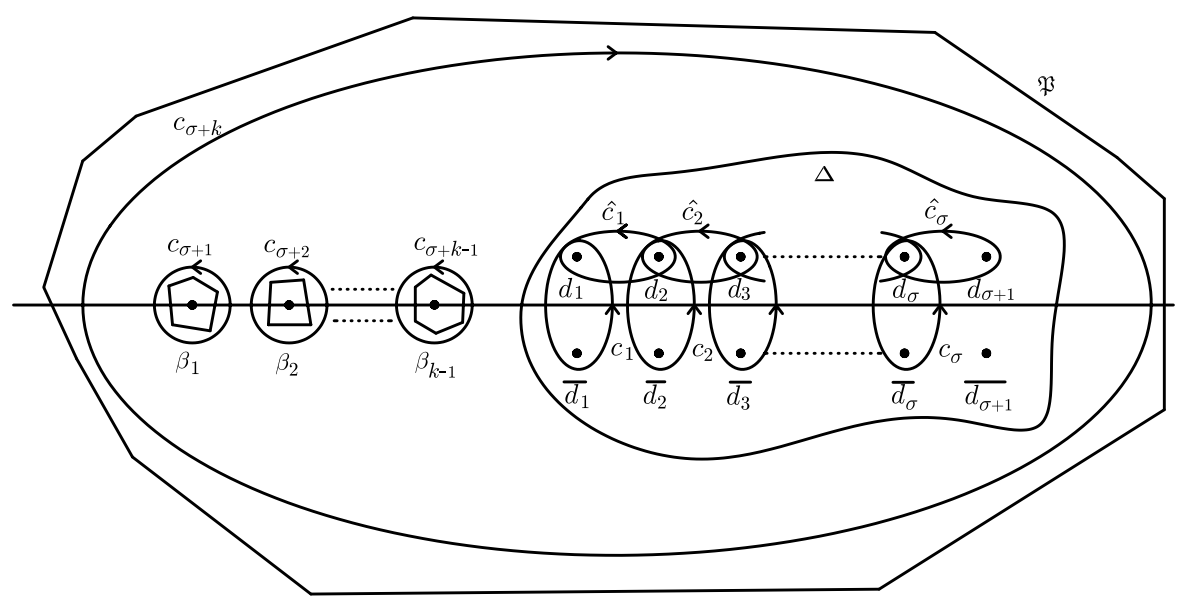

Figure 2. The $z$-projection of the curves in $\mathcal{B}$.

To prove our theorem we will need to close the period problem for holomorphic 1-forms on $D(\mathfrak{P}), \Phi$, satisfying $I^{*}(\Phi)=\bar{\Phi}$. In this case we have the following claim.

Claim 1. If $\tau$ is a holomorphic differential on $D(\mathfrak{P})$ satisfying $I^{*}(\tau)=\bar{\tau}$, then $\operatorname{Re}\left(\int_{\gamma} \tau\right)=0, \forall \gamma \in H_{1}(D(\mathfrak{P}), \mathbb{R})$ if, and only if, $\int_{\gamma_{j}} \tau=0, j=1, \ldots, \sigma+k$.

In particular, if in addition $\tau$ is holomorphic in $\bar{M}$, then $\tau=0$ if, and only if, $\int_{\gamma_{j}} \tau=0, j=1, \ldots, \sigma$.

Proof. Observe that $\int_{\gamma} \tau=\overline{\int_{I_{*}(\gamma)} \tau}$. From the choice of $\mathcal{B}$, the first part of the claim trivially holds. For the second part, take into account that a holomorphic differential on $\bar{M}$ vanishes if, and only if, it has imaginary periods.

We denote $\mathcal{V}=\{\omega$ holomorphic differentials on $M\}$. It is clear that $\omega \in \mathcal{V}$ if, and only if, $\overline{I^{*} \omega} \in \mathcal{V}$. Define the following real subspaces of $\mathcal{V}$ :

$$
\mathcal{V}^{+}=\left\{\tau \in \mathcal{V} / I^{*} \tau=\bar{\tau}\right\}, \quad \mathcal{V}^{-}=\left\{\tau \in \mathcal{V} / I^{*} \tau=-\bar{\tau}\right\} .
$$


Claim 2. Let $p$ be a point in $M$ and $\omega \in \mathcal{V}^{-}$satisfying $A^{*} \omega=-\omega$. Then it is possible to construct a holomorphic function $H: M \longrightarrow \mathbb{C}$ such that:

(i) $H \circ I=-\bar{H}, H \circ A=-H$,

(ii) $(\omega+d H)_{0} \geq(\omega)_{0} \cdot p \cdot I(p) \cdot A(p) \cdot I(A(p))$.

Proof. From the hypotheses of the claim we know that $\omega=\frac{P(z)}{Q(z)} \frac{d z}{w}$, where $P(z)$ and $Q(z)$ are polynomial with real coefficients and the roots of $Q$ belong to the set $\left\{\beta_{1}, \ldots, \beta_{k-1}\right\}$. Assume that $(\omega)_{0}=p^{n} \cdot I(p)^{n} \cdot A(p)^{n} \cdot I(A(p))^{n} \cdot E, n \geq 0$, where $E$ is an integral divisor not containing the points $p, I(p), A(p)$ and $I(A(p))$. Define:

$$
J(z, w)= \begin{cases}\frac{P(z)^{2}}{(z-z(p))^{n-1}(z-\overline{z(p)})^{n-1}} w, & \text { if } p \neq A(p) \text { and } p \neq I(A(p)), \\ \frac{P(z)^{2}}{(z-z(p))^{n-1}} w, & \text { if } p \neq A(p) \text { and } p=I(A(p)), \\ \frac{P(z)^{2}}{(z-z(p))^{n}(z-\overline{z(p)})^{n}} w, & \text { if } p=A(p) .\end{cases}
$$

It is clear that $J$ satisfies (i) and that it has zeroes at $p, I(p), A(p)$ and $I(A(p))$ of order $n+1$. Furthermore the divisor $E^{2}$ is contained in $(J)_{0}$. Then it is possible to find $\lambda \in \mathbb{R}$ such that $(\omega+\lambda d J)_{0} \geq(\omega)_{0} \cdot p \cdot I(p) \cdot A(p) \cdot I(A(p))$. This completes the proof.

Claim 3. Consider $\left(b_{1}, \ldots, b_{\sigma+k}\right) \in \mathbb{R}^{\sigma+k}-\{(0, \ldots, 0)\}$ and $c=\sum_{j=1}^{\sigma+k} b_{j} \gamma_{j}$. Then there exists $\tau \in \mathcal{V}^{-}$satisfying $\operatorname{Im}\left(\int_{c} \tau\right) \neq 0$.

Furthermore, if $L$ is an integral divisor invariant under I and whose support is in $D(\mathfrak{P})$, then $\tau$ can be chosen in such a way that $(\tau)_{0} \geq L$.

Proof. We consider the linear map $f: \mathcal{V}^{-} \rightarrow \mathbb{R}^{\sigma+k}, f(\omega)=\left(\int_{\gamma_{j}}-i \omega\right)_{j=1, \ldots, \sigma+k}$. Note that the following differentials are linearly independent and are not in the kernel of $f$ :

$$
\left\{z^{m} \frac{d z}{w}, \quad m=0, \ldots, \sigma\right\} \cup\left\{\frac{d z}{\left(z-\beta_{m}\right) w}, \quad m=1, \ldots, k-1\right\} .
$$

Using Claim 1 we get that the matrix

$$
\left(f\left(z^{m} \frac{d z}{w}\right)\right)_{m=0, \ldots, \sigma-1}=\left(a_{0}^{m}, \ldots, a_{\sigma-1}^{m}, 0, \ldots, 0\right)_{m=0, \ldots, \sigma-1}
$$

has range $\sigma$. Moreover, it is easy to check that $f\left(\frac{d z}{\left(z-\beta_{j}\right) w}\right)=\left(a_{0}^{j}, \ldots, a_{\sigma-1}^{j}, 0, \ldots, 0\right.$, $\left.2^{j} \pi b_{j}, 0, \ldots, 0\right)$, and $f\left(z^{\sigma} \frac{d z}{w}\right)=\left(a_{0}^{k}, \ldots, a_{\sigma-1}^{k}, 0, \ldots, 0,2 \pi b_{k}\right)$, where $b_{j} \in \mathbb{R}^{*}, j=$ $1, \ldots, k$. Both facts imply that $f$ is onto. Thanks to this there exists $\tau_{0} \in \mathcal{V}^{-}$such that $\operatorname{Im}\left(\int_{c} \tau_{0}\right) \neq 0$.

Moreover, taking into account our construction, we know that $A^{*}\left(\tau_{0}\right)=-\tau_{0}$. The second part of the lemma is a consequence of a successive use of Claim 2

Claim 4. Let $H^{-}(\overline{D(\mathfrak{P})})$ be the real vector space of the holomorphic functions $t$ : $\overline{D(\mathfrak{P})} \rightarrow \mathbb{C}$ satisfying $t \circ I=-\bar{t}$. Then the linear map $F: H^{-}(\overline{D(\mathfrak{P})}) \rightarrow \mathbb{R}^{2(\sigma+k)}$, 
given by

$$
F(t)=\left(\int_{\gamma_{j}} t \Phi_{3}\left(\frac{1}{g}+g\right),-i \int_{\gamma_{j}} t \Phi_{3}\left(\frac{1}{g}-g\right)\right)_{j=1, \ldots, \sigma+k},
$$

is surjective.

Proof. We proceed by contradiction. Assume $F$ is not onto. Then, there is

$$
\left(\vartheta_{1}, \ldots, \vartheta_{\sigma+k}, \mu_{1}, \ldots, \mu_{\sigma+k}\right) \in \mathbb{R}^{2(\sigma+k)}-\{(0, \ldots, 0)\},
$$

such that

(2) $\sum_{j=1}^{\sigma+k}\left[\vartheta_{j} \int_{\gamma_{j}} t \Phi_{3}\left(\frac{1}{g}+g\right)-i \mu_{j} \int_{\gamma_{j}} t \Phi_{3}\left(\frac{1}{g}-g\right)\right]=0 \quad \forall t \in H^{-}(\overline{D(\mathfrak{P})})$.

Claim 3 guarantees the existence of a differential $\tau \in \mathcal{V}^{-}$satisfying

(i) $(\tau)_{0} \geq\left(\left(\left(\frac{1}{g}+g\right) \Phi_{3}\right)_{\left.\right|_{\bar{\Omega}}}\right)_{0}^{2}\left(\left(d\left(\frac{1-g^{2}}{1+g^{2}}\right)\right)_{\left.\right|_{\bar{\Omega}}}\right)_{0}$,

(ii) $-i \sum_{j=1}^{\sigma+k} \mu_{j} \int_{\gamma_{j}} \tau \neq 0$.

Let us define $y \stackrel{\text { def }}{=} \frac{\tau}{d\left(\frac{1-g^{2}}{1+g^{2}}\right)}$ and $t \stackrel{\text { def }}{=} \frac{d(y)}{\left(\frac{1}{g}+g\right) \Phi_{3}}$. Taking the choice of $\tau$ into account the function $t$ belongs to $H^{-}(\overline{D(\mathfrak{P})})$. In this case, and integrating by parts, (2) becomes $-i \sum_{j=1}^{\sigma+k} \mu_{j} \int_{\gamma_{j}} \tau=0$, which is absurd. This contradiction proves the claim.

Using the previous claim we infer the existence of $\left\{t_{1}, \ldots, t_{2(\sigma+k)}\right\} \subset H^{-}(\overline{D(\mathfrak{P})})$ such that $\operatorname{det}\left(F\left(t_{1}\right), \ldots, F\left(t_{2(\sigma+k)}\right)\right) \neq 0$. Up to changing $t_{i} \leftrightarrow t_{i} / x, x>0$, large enough, we can assume that

$$
\left|\exp \left(\sum_{i=1}^{2(\sigma+k)} x_{i} t_{i}(p)\right)-1\right|<1 /(2 \alpha),
$$

$\forall\left(x_{1}, \ldots, x_{2(\sigma+k)}\right) \in \mathbb{R}^{2(\sigma+k)},\left|x_{i}\right|<1, i=1, \ldots, 2(\sigma+k), \quad \forall p \in \overline{D(\mathfrak{P})}$.

Claim 5. For each $n \in \mathbb{N}$, there is $t_{0}^{n} \in H^{-}(\overline{D(\mathfrak{P})})$ such that:

(i) $\left|t_{0}^{n}-n \log \alpha\right|<1 / n$ in $K_{1}$ (and so $\left|t_{0}^{n}+n \log \alpha\right|<1 / n$ in $I\left(K_{1}\right)$ ),

(ii) $\left|t_{0}^{n}\right|<1 / n$ in $K_{2}$.

Proof. Given $n \in \mathbb{N}$, we apply a Runge-type theorem on $\bar{M}$ (see [8, Theorem 10]) and obtain a holomorphic function $T_{0}^{n}: \overline{D(\mathfrak{P})} \rightarrow \mathbb{C}$ satisfying

- $\left|T_{0}^{n}-n \log \alpha\right|<1 / n$ in $K_{1}$,

- $\left|T_{0}^{n}+n \log \alpha\right|<1 / n$ in $I\left(K_{1}\right)$,

- $\left|T_{0}^{n}\right|<1 / n$ in $K_{2}$.

We take $t_{0}^{n}=\frac{1}{2}\left(T_{0}^{n}-\overline{T_{0}^{n} \circ I}\right)$. From this, it is trivial to check properties (i) and (ii). 
For $\Theta=\left(\lambda_{0}, \ldots, \lambda_{2(\sigma+k)}\right) \in \mathbb{R}^{2(\sigma+k)+1}$, we define

$$
h^{\Theta, n}(p) \stackrel{\text { def }}{=} \exp \left[\lambda_{0} t_{0}^{n}(p)+\sum_{j=1}^{2(\sigma+k)} \lambda_{j} t_{j}(p)\right], \quad \forall p \in \overline{D(\mathfrak{P})} .
$$

Label $g^{\Theta, n}=g / h^{\Theta, n}$ and $\Phi_{3}^{\Theta, n}=\Phi_{3}$. As $\left\{t_{\left.0\right|_{K_{2}}}^{n}\right\}_{n \in \mathbb{N}}$ is uniformly bounded, then, up to a subsequence, we have $\left\{t_{\left.0\right|_{K_{2}}}^{n}\right\} \rightarrow t_{0}^{\infty} \equiv 0$, uniformly on $K_{2}$. We also define on $K_{2}$ the Weierstrass data $g^{\Theta, \infty}=g / h^{\Theta, \infty}, \Phi_{3}^{\Theta, \infty}=\Phi_{3}$, where

$$
h^{\Theta, \infty}(p) \stackrel{\text { def }}{=} \exp \left[\sum_{j=1}^{2(\sigma+k)} \lambda_{j} t_{j}(p)\right], \quad \forall p \in K_{2} .
$$

Observe that the third Weierstrass differential of the aforementioned holomorphic data has no real periods. Then we must only consider the period problem associated to $\Phi_{j}^{\Theta, n}, j=1,2$. To do this, we define the period map $\mathcal{P}_{n}: \mathbb{R}^{2(\sigma+k)+1} \rightarrow \mathbb{R}^{2(\sigma+k)}$, $n \in \mathbb{N} \cup\{\infty\}:$

$$
\mathcal{P}_{n}(\Theta)=\left(\int_{\gamma_{j}} \Phi_{1}^{\Theta, n}, \int_{\gamma_{j}} \Phi_{2}^{\Theta, n}\right)_{j=1, \ldots, \sigma+k} .
$$

Since the initial immersion $X$ is well defined, one has $\mathcal{P}_{n}(0)=0, \forall n \in \mathbb{N} \cup\{\infty\}$. Moreover, it is not hard to check that

$$
\operatorname{Jac}_{\lambda_{1}, \ldots, \lambda_{2(\sigma+k)}}\left(\mathcal{P}_{n}\right)(0)=\operatorname{det}\left(F\left(t_{1}\right), \ldots, F\left(t_{2(\sigma+k)}\right)\right) \neq 0, \quad \forall n \in \mathbb{N} \cup\{\infty\} .
$$

Applying the Implicit Function Theorem to the map $\mathcal{P}_{n}$ at $0 \in[-\epsilon, \epsilon] \times \bar{B}(0, r)$, we get a smooth function $L_{n}: I_{n} \rightarrow \mathbb{R}^{2(\sigma+k)}$ satisfying $\mathcal{P}_{n}\left(\lambda_{0}, L_{n}\left(\lambda_{0}\right)\right)=0, \forall \lambda_{0} \in$ $I_{n}$, where $I_{n}$ is a maximal open interval containing 0 (here, maximal means that $L_{n}$ cannot be regularly extended beyond $I_{n}$ ).

Let us see that the supremum $\epsilon_{n}$ of the connected component of $L_{n}^{-1}(\bar{B}(0, r)) \cap$ $[0, \epsilon]$ containing $\lambda_{0}=0$ belongs to $I_{n}$. Indeed, take a sequence $\left\{\lambda_{0}^{k}\right\}_{k \in \mathbb{N}} \nearrow \epsilon_{n}$. As $\left\{L_{n}\left(\lambda_{0}^{k}\right)\right\} \subset \bar{B}(0, r)$, then, up to a subsequence, $\left\{L_{n}\left(\lambda_{0}^{k}\right)\right\}_{k \in \mathbb{N}} \rightarrow \Lambda_{n} \in \bar{B}(0, r)$. Taking into account that $\operatorname{Jac}_{\lambda_{1}, \ldots, \lambda_{2(\sigma+k)}}\left(\mathcal{P}_{n}\right)\left(\epsilon_{n}, \Lambda_{n}\right) \neq 0$, the local unicity of the curve $\left(\lambda_{0}, L_{n}\left(\lambda_{0}\right)\right)$ around the point $\left(\epsilon_{n}, \Lambda_{n}\right)$, and the maximality of $I_{n}$, we infer that $\epsilon_{n} \in I_{n}$. Therefore, either $\epsilon_{n}=\epsilon$ or $L_{n}\left(\epsilon_{n}\right)=\Lambda_{n} \in \partial(B(0, r))$.

We will now see that $\epsilon_{0} \stackrel{\text { def }}{=} \liminf \left\{\epsilon_{n}\right\}>0$. Otherwise, there would be a subsequence $\left\{\epsilon_{n}\right\} \rightarrow 0$. Without loss of generality, $\epsilon_{n}<\epsilon, \forall n \in \mathbb{N}$, and so $\Lambda_{n} \in \partial(B(0, r)), \forall n \in \mathbb{N}$. Up to a subsequence, $\left\{\Lambda_{n}\right\} \rightarrow \Lambda_{\infty} \in \partial(B(0, r))$. The fact $\mathcal{P}_{\infty}(0,0)=\mathcal{P}_{\infty}\left(0, \Lambda_{\infty}\right)=0$ would contradict the injectivity of $\mathcal{P}_{\infty}(0, \cdot)$ in $\bar{B}(0, r)$. Hence the function $L_{n}:\left[0, \epsilon_{0}\right] \rightarrow \bar{B}(0, r)$ is well defined, $\forall n \geq n_{0}, n_{0}>1 / \epsilon_{0}, n_{0}$ large enough.

Label $\left(\lambda_{1}^{n}, \ldots, \lambda_{2(\sigma+k)}^{n}\right)=L_{n}(1 / n)$. From (3) we have $\left|\exp \left[\sum_{j=1}^{2(\sigma+k)} \lambda_{j}^{n} t_{j}\right]-1\right|<$ $1 /(2 \alpha)$ on $D(\overline{\mathfrak{P}})$. Hence, if $n\left(\geq n_{0}\right)$ is large enough, the function

$$
h(z) \stackrel{\text { def }}{=} \exp \left[\frac{1}{n} t_{0}^{n}(z)+\sum_{j=1}^{2(\sigma+k)} \lambda_{j}^{n} t_{j}(z)\right]
$$


satisfies statements 1, 2 and 3 in Lemma 1. As the period function $\mathcal{P}_{n}$ vanishes at $\Theta_{n}=\left(1 / n, \lambda_{1}^{n}, \ldots, \lambda_{2(\sigma+k)}^{n}\right)$, then the minimal immersion $\widetilde{F}$ associated to the Weierstrass data $g^{\Theta_{n}, n}, \Phi_{3}^{\Theta_{n}, n}=\Phi_{3}$ is well defined. This proves statement 4 in the lemma.

Proof of Lemma 2. Consider $\mathfrak{P}$, the multigon given in the statement of the lemma, $\mathfrak{P}=\left\{P_{1}, P_{2}, \ldots, P_{k}\right\}$. As usual in this kind of construction, we are going to follow 2 to describe a labyrinth on $D(\mathfrak{P}) \subset M$ depending on $\mathfrak{P}$ and a positive integer $N$. Then, we use Lemma 1 following Nadirashvili's ideas [5].

From now on, $N$ will represent a positive multiple of $k$, and we will always assume that $N$ is large enough to satisfy all the inequalities where it appears. In what follows, the symbol const will denote a family of positive real constants that do not depend on $N$. These constants only depend on $X, \mathfrak{P}, r, \varepsilon$ and $s$. So, we will use this symbol to refer distinct constants that appear in different parts of the proof.

Let $v_{1, j}, \ldots, v_{\frac{2 N}{k}, j}$ be a set of points in the polygon $P_{j}$ (containing the vertices of $\left.P_{j}\right)$ that divide each side of the polygon $P_{j}$ into $2 N /\left(k l_{i}\right)$ segments of equal length, where $l_{j}$ is the number of sides of $P_{j}$. We are supposing that $N$ is a multiple of $l_{1}, \ldots, l_{k}$. We transfer this partition to the parallel polygon $P_{j}^{2 / N}: v_{1, j}^{\prime}, \ldots, v_{2 N / k, j}^{\prime}$. Define the following sets:

- $L_{i, j}=$ the segment that joins $v_{i, j}$ and $v_{i, j}^{\prime}, i=1, \ldots 2 N / k$;

- $P_{i, j}=P_{j}^{i / N^{3}}, i=0, \ldots, 2 N^{2}$ (recall that $P_{j}^{i / N^{3}}$ means the parallel polygon to $P_{j}$, in $\operatorname{Int}(\mathfrak{P})$, such that the distance between parallel sides is $\left.i / N^{3}\right)$;

- If $j<k, \quad \mathcal{A}_{j}=\bigcup_{i=0}^{N^{2}-1} \overline{\operatorname{Int}\left(P_{2 i+1, j}\right) \backslash \operatorname{Int}\left(P_{2 i, j}\right)}$ and $\widetilde{\mathcal{A}}_{j}=\bigcup_{i=1}^{N^{2}} \overline{\operatorname{Int}\left(P_{2 i, j}\right) \backslash \operatorname{Int}\left(P_{2 i-1, j}\right)}$;

- If $j=k, \quad \mathcal{A}_{k}=\bigcup_{i=0}^{N^{2}-1} \overline{\operatorname{Int}\left(P_{2 i, k}\right) \backslash \operatorname{Int}\left(P_{2 i+1, k}\right)}$ and $\widetilde{\mathcal{A}}_{k}=\bigcup_{i=1}^{N^{2}} \overline{\operatorname{Int}\left(P_{2 i-1, k}\right) \backslash \operatorname{Int}\left(P_{2 i, k}\right)}$;

- $\mathcal{R}_{j}=\bigcup_{i=0}^{2 N^{2}} P_{i, j}$;

- $L_{j}=\bigcup_{i=1}^{N / k} L_{2 i, j} \cap \mathcal{A}_{j}, \widetilde{L}_{j}=\bigcup_{i=0}^{N / k-1} L_{2 i+1, j} \cap \widetilde{\mathcal{A}}_{j}$, and $H_{j}=\mathcal{R}_{j} \cup L_{j} \cup \widetilde{L}_{j}$;

- If $j<k, \quad \Omega_{N, j}=\left\{z \in \operatorname{Int}\left(P_{2 N^{2}, j}\right) \backslash \operatorname{Int}\left(P_{0, j}\right): \operatorname{dist}_{\left(d s_{0}, \mathbb{C}\right)}\left(z, H_{j}\right) \geq \frac{1}{4 N^{3}}\right\}$;

- If $j=k, \quad \Omega_{N, k}=\left\{z \in \operatorname{Int}\left(P_{0, k}\right) \backslash \operatorname{Int}\left(P_{2 N^{2}, k}\right): \operatorname{dist}_{\left(d s_{0}, \mathbb{C}\right)}\left(z, H_{k}\right) \geq \frac{1}{4 N^{3}}\right\}$; where $d s_{0}$ is the Euclidean metric on $\mathbb{C}$;

- $\Omega_{N}=\bigcup_{j=1}^{k} \Omega_{N, j}$.

We define $V_{i, j}$ as the union of the segment $L_{i, j}$ and those connected components of $\Omega_{N, j}$ that have nonempty intersection with $L_{i, j}$ for $i=1, \ldots, 2 N / k$. We define the sets $\omega_{l}, l=1, \ldots, 2 N$, as $\omega_{(j-1)} \frac{2 N}{k}+i=V_{i, j}, i=1, \ldots, 2 N / k, j=1, \ldots, k$. We label $\varpi_{i}=\left\{z \in \mathbb{C}: \operatorname{dist}_{\left(d s_{0}, \mathbb{C}\right)}\left(z, \omega_{i}\right)^{k}<\delta(N)\right\}$, where $i=1, \ldots, 2 N$, and $\delta(N)>0$ is chosen in such a way that the sets $\bar{\varpi}_{i}(i=1, \ldots, 2 N)$ are pairwise disjoint (see Figure [3). Finally, we denote $\left.\omega_{i}^{\prime} \stackrel{\text { def }}{=} f_{+}\left(\omega_{i}\right), \varpi_{i}^{\prime} \stackrel{\text { def }}{=} f_{+}\left(\varpi_{i}\right), \omega_{i}^{*} \stackrel{\text { def }}{=} \omega_{i}^{\prime} \cup I\left(\omega_{i}^{\prime}\right)\right)$, and $\varpi_{i}^{*}=\varpi_{i}^{\prime} \cup I\left(\varpi_{i}^{\prime}\right)$.

Now, we consider the Riemannian metric $d s^{2}=\|d z / w\|^{2}$ on $M$. Observe that the metrics $d s^{2}$ and $\|d z\|$ are equivalent in $\overline{D(\mathfrak{P})} \backslash D\left(\mathfrak{P}^{\varepsilon}\right)$, i.e.,

$$
\text { const }\left\|\frac{d z}{w}\right\| \leq\|d z\| \leq \text { const }\left\|\frac{d z}{w}\right\| \quad \text { in } \overline{D(\mathfrak{P})} \backslash D\left(\mathfrak{P}^{\varepsilon}\right) \text {. }
$$




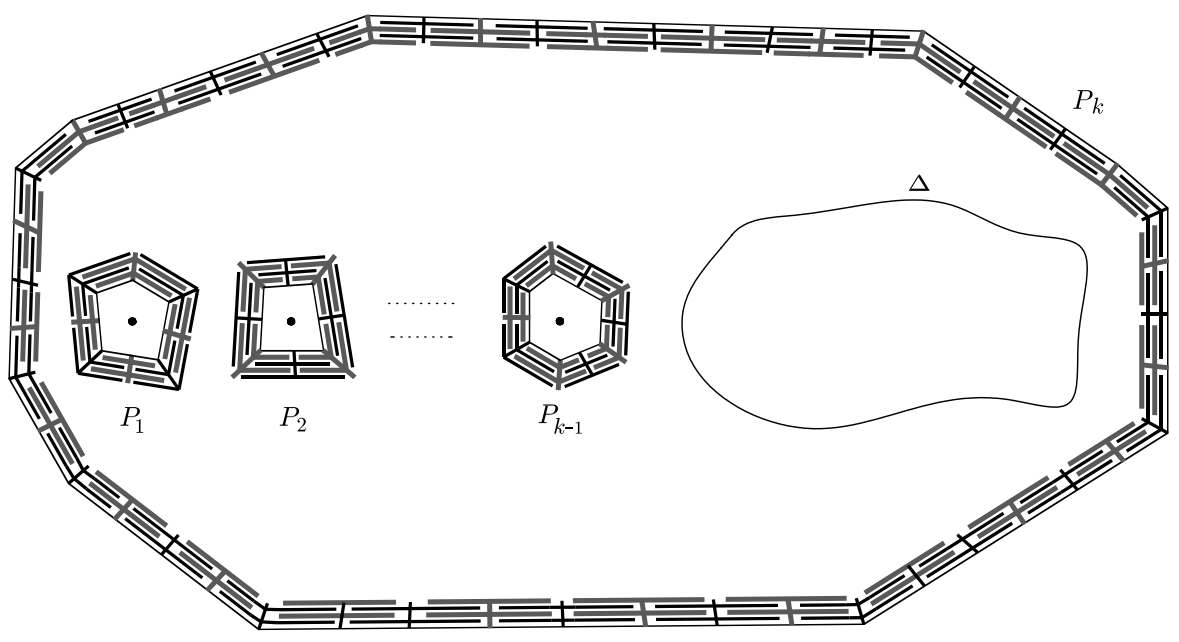

Figure 3. The labyrinth.

Thanks to this relation, we can translate some metric properties of the sets $\varpi_{i}$ to the sets $\varpi_{i}^{*}$. If $N$ is large enough, it is clear that we can guarantee the following properties:

Claim A. $\operatorname{diam}_{d s}\left(\varpi_{i}^{j}\right) \leq \frac{\text { const }}{N}, j=1,2$.

Claim B. If $\lambda^{2}(z, w) d s^{2}$ is a conformal metric in $\overline{D(\mathfrak{P})}$ and $\Upsilon \in \mathbb{R}^{+}$satisfying

$$
\lambda(z, w) \geq \begin{cases}\Upsilon & \text { in } \overline{D(\mathfrak{P})}, \\ \Upsilon N^{4} & \text { in } \bigcup_{j=1}^{2 N} \omega_{j}^{*},\end{cases}
$$

and if $\alpha$ is a curve in $D(\mathfrak{P})$ connecting $\partial\left(D\left(\mathfrak{P}^{\varepsilon}\right)\right)$ and $\partial(D(\mathfrak{P}))$, then length $(\alpha, \lambda d s)$ $\geq$ const $\Upsilon N$, where const does not depend on $\Upsilon$.

Our next stage involves a modification of the immersion $X$ in $2 N$ steps, where each step is related to each set $\varpi_{i}^{*}$. This modification consists of constructing a sequence of $2 N$ conformal minimal immersions $F_{i}: \overline{D(\mathfrak{P})} \rightarrow \mathbb{R}^{3}, i=0,1, \ldots, 2 N$, with $F_{0}=X$, in a recursive way. The immersion obtained in the last step, $F_{2 N}$, will be the immersion that proves the lemma. In order to get this, the sequence of immersions must satisfy the following properties:

$\left(\mathrm{P} 1_{i}\right) F_{i}(p)=\operatorname{Re}\left(\int_{p_{0}}^{p} \Phi^{i}\right)$, where $I^{*} \Phi^{i}=\overline{\Phi^{i}}$, $\Phi^{i}=\left(\varphi_{1}^{i}(z, w), \varphi_{2}^{i}(z, w), \varphi_{3}^{i}(z, w)\right) \frac{d z}{w}$

$\left(\mathrm{P} 2_{i}\right)\left\|\varphi^{i}-\varphi^{i-1}\right\| \leq 1 / N^{2}$ in $D(\mathfrak{P}) \backslash \varpi_{i}^{*}$;

$\left(\mathrm{P} 3_{i}\right)\left\|\varphi^{i}\right\| \geq N^{7 / 2}$ in $\omega_{i}^{*}$;

$\left(\mathrm{P} 4_{i}\right)\left\|\varphi^{i}\right\| \geq \frac{\text { const }}{\sqrt{N}}$ in $\varpi_{i}^{*}$;

$\left(\mathrm{P} 5_{i}\right) \operatorname{dist}_{\left(d s_{1}, \mathbb{S}^{2}\right)}\left(\mathcal{G}_{i}(z, w), \mathcal{G}_{i-1}(z, w)\right)<\frac{1}{N \sqrt{N}}$ in $D(\mathfrak{P}) \backslash \varpi_{i}^{*}$, where $d s_{1}$ is the usual Riemannian metric in $\mathbb{S}^{2}$ and $\mathcal{G}_{i}$ represents the Gauss map of the immersion $F_{i}$; 
$\left(\mathrm{P} 6_{i}\right)$ there exists an orthogonal frame $S_{i}=\left\{e_{1}, e_{2}, e_{3}\right\}$ in $\mathbb{R}^{3}$ such that:

(P6.1 $\left.1_{i}\right)$ if $p \in \overline{\varpi_{i}^{*}}$ and $\left\|F_{i-1}(p)\right\| \geq 1 / \sqrt{N}$, then

$$
\left\|\left(F_{i-1}(p)\right)_{*}\right\|<\frac{\text { const }}{\sqrt{N}}\left\|F_{i-1}(p)\right\|,
$$

$\left(\mathrm{P} 6.2_{i}\right)\left(F_{i}(p)\right)_{3}=\left(F_{i-1}(p)\right)_{3}$ for all $p \in \overline{D(\mathfrak{P})}$,

where $(\cdot)_{k}$ is the $k$ th coordinate function and $(\cdot)_{*}=\left((\cdot)_{1},(\cdot)_{2}\right)$, with respect to $S_{i}=\left\{e_{1}, e_{2}, e_{3}\right\}$.

Now, we construct the above sequence of immersions in a recursive way. Suppose that we have $F_{0}, \ldots, F_{j-1}$ satisfying the above claims. We must define $F_{j}$.

Observe that for an $N$ large enough, the immersion $F_{j-1}$ satisfies the following properties:

(L1) $\left\|\varphi^{j-1}\right\| \leq$ const in $D(\mathfrak{P}) \backslash \bigcup_{l=1}^{j-1} \varpi_{l}^{*}$.

(L2) $\left\|\varphi^{j-1}\right\| \geq$ const in $D(\mathfrak{P}) \backslash \bigcup_{l=1}^{j-1} \varpi_{l}^{*}$.

(L3) $F_{j-1} \circ I=F_{j-1}$ and $\mathcal{G}_{j-1} \circ I=-\mathcal{G}_{j-1}$.

(L4) The diameter in $\mathbb{S}^{2}$ of $\mathcal{G}_{j-1}\left(\varpi_{j}^{\prime}\right)$ is less than $\frac{\text { const }}{\sqrt{N}}$, and the diameter in $\mathbb{R}^{3}$ of $F_{j-1}\left(\varpi_{j}^{\prime}\right)$ is less than $\frac{\text { const }}{N}$.

(L5) There is a set of orthogonal coordinates $S_{j}=\left\{e_{1}, e_{2}, e_{3}\right\}$ in $\mathbb{R}^{3}>0$ such that:

(L5.a) if $p \in \varpi_{j}^{*}$ and $\left\|F_{j-1}(p)\right\| \geq \frac{1}{\sqrt{N}}$, then $\angle\left(e_{3}, F_{j-1}(p)\right) \leq \frac{\text { const }}{\sqrt{N}}$,

(L5.b) $\angle\left( \pm e_{3}, \mathcal{G}_{j-1}\right) \geq \frac{\text { const }}{\sqrt{N}}$ in $\varpi_{j}^{*}$.

Here, $\angle(a, b) \in\left[0, \pi\left[\right.\right.$ is the angle formed by $a$ and $b$ in $\mathbb{R}^{3}$.

Claims (L1)-(L4) easily deduce from the properties ( $\left.\mathrm{P}_{-}\right)$. The choice of the coordinates $S_{j}$ in (L5) is possible as a consequence of the bounds of the diameters of $F_{j-1}\left(\varpi_{j}^{l}\right)$ and $\mathcal{G}_{j-1}\left(\varpi_{j}^{l}\right)$ given in the statement of (L3) and property (L4).

We shall now construct $F_{j}$. Let $\left(\Phi_{3}^{j-1}, g^{j-1}\right)$ be the Weierstrass data of the immersion $F_{j-1}$ in the coordinate system $S_{j}$. Let $h_{\alpha}$ be the function given by Lemma 1 for $K_{1}=\omega_{j}^{\prime}, K_{2}=\overline{D(\mathfrak{P})} \backslash \varpi_{j}^{*}$ and $\alpha$ large enough in terms of $N$. We define the minimal immersion $F_{j}$ in the frame $S_{j}$ as $F_{j}(p)=\operatorname{Re}\left(\int_{p_{0}}^{p} \Phi^{j}\right)$, where $\Phi^{j}$ is determined by $\Phi_{3}^{j}=\Phi_{3}^{j-1}$ and $g^{j}=g^{j-1} / h_{\alpha}$. We denote $\Phi^{j}=\varphi^{j} \frac{d z}{w}$. Observe that $F_{j}$ is well defined because Lemma 1 tells us that the Weierstrass data $\Phi^{j}$ has no real periods.

We shall now see that $F_{j}$ satisfies properties $\left(\mathrm{P} 1_{j}\right)-\left(\mathrm{P} 6_{j}\right)$. Claim $\left(\mathrm{P} 1_{j}\right)$ easily holds. Claims $\left(\mathrm{P} 2_{j}\right)$ and $\left(\mathrm{P} 5_{j}\right)$ are a consequence of the fact that $h_{\alpha} \rightarrow 1$ uniformly on $K_{2}=\overline{D(\mathfrak{P})} \backslash \varpi_{j}^{*}$ as $\alpha \rightarrow \infty$. To satisfy $\left(\mathrm{P} 3_{j}\right)$, we take into account that $h_{\alpha} \rightarrow \infty$ uniformly on $K_{1}=\omega_{j}^{\prime}$ as $\alpha \rightarrow \infty$. So, $\left\|\Phi^{j}\right\| \geq N^{7 / 2}$ in $\omega_{j}^{\prime}$, for $\alpha$ large enough. Property $\left(\mathrm{P} 3_{j}\right)$ is a consequence of $I^{*} \Phi_{j}=\overline{\Phi_{j}}$. To deduce property $\left(\mathrm{P} 4_{j}\right)$, we need the next inequalities:

$$
\frac{\sin \left(\frac{\text { const }}{\sqrt{N}}\right)}{1+\cos \left(\frac{\text { const }}{\sqrt{N}}\right)} \leq\left|g^{j-1}\right| \leq \frac{\sin \left(\frac{\text { const }}{\sqrt{N}}\right)}{1-\cos \left(\frac{\text { const }}{\sqrt{N}}\right)} \quad \text { in } \varpi_{j}^{*} .
$$

These inequalities are due to (L5.b). Then using (L2) and (4) (for a large enough $N)$, one has

$$
\left\|\varphi^{j}\right\| \geq\left|\varphi_{3}^{j}\right|=\left|\varphi_{3}^{j-1}\right| \geq \sqrt{2}\left\|\varphi^{j-1}\right\| \frac{\left|g^{j-1}\right|}{1+\left|g^{j-1}\right|^{2}} \geq \text { const } \sin \left(\frac{\text { const }}{\sqrt{N}}\right) \geq \frac{\text { const }}{\sqrt{N}} \quad \text { in } \varpi_{j}^{*} .
$$

To obtain $\left(\mathrm{P} 6.1_{j}\right)$ and $\left(\mathrm{P} 6.2_{j}\right)$, use that $\Phi_{3}^{j-1}=\Phi_{3}^{j}$ in the frame $S_{j}$, and (L5.a). 
Hence, we have constructed the immersions $F_{0}, F_{1}, \ldots, F_{2 N}$ satisfying claims $\left(\mathrm{P} 1_{j}\right)-\left(\mathrm{P} 6_{j}\right)$ for $j=1, \ldots, 2 N$.

In the following proposition, we study the properties of the immersion $F_{2 N}$.

Proposition 1. If $N$ is large enough, then $F_{2 N}$ satisfies that:

(i) $2 s<\operatorname{dist}_{\left(F_{2 N}, \overline{D(\mathfrak{P}))}\right.}\left(\partial(D(\mathfrak{P})), \partial\left(D\left(\mathfrak{P}^{\epsilon}\right)\right)\right)$;

(ii) $\left\|F_{j}-F_{j-1}\right\| \leq \frac{\text { const }}{N^{2}}$ in $D(\mathfrak{P}) \backslash \varpi_{j}^{*}$;

(iii) $\left\|F_{2 N}-X\right\| \leq \frac{\text { const }}{N}$ in $D(\mathfrak{P}) \backslash \bigcup_{j=1}^{2 N} \varpi_{j}^{*}$;

(iv) there is a multigon $\widetilde{\mathfrak{P}}$ satisfying:

(iv.1) $\overline{\operatorname{Int}\left(\mathfrak{P}^{\varepsilon}\right)} \subset \operatorname{Int}(\widetilde{\mathfrak{P}}) \subset \overline{\operatorname{Int}(\widetilde{\mathfrak{P}})} \subset \operatorname{Int}(\mathfrak{P})$;

(iv.2) $s<\operatorname{dist}_{\left(F_{2 N}, \overline{D(\tilde{\mathfrak{P}}))}\right.}\left(p, \partial\left(D\left(\mathfrak{P}^{\varepsilon}\right)\right)\right)<2 s, \forall p \in \partial(D(\widetilde{\mathfrak{P}}))$;

(iv.3) $F_{2 N}(D(\widetilde{\mathfrak{P}})) \subset B_{R}$, where $R=\sqrt{r^{2}+(2 s)^{2}}+\varepsilon$.

Proof. Properties (L2), $\left(\mathrm{P} 2_{i}\right),\left(\mathrm{P} 3_{i}\right)$ and $\left(\mathrm{P} 4_{i}\right)$ give us the following bound of the conformal metric $\lambda_{F_{2 N}}^{2}(z)\left\|\frac{d z}{w}\right\|^{2}$ :

$$
\lambda_{F_{2 N}}(z, w) \geq \begin{cases}\Upsilon & \text { in } \overline{D(\mathfrak{P})}, \\ \Upsilon N^{4} & \text { in } z^{-1}\left(\Omega_{N}\right)\end{cases}
$$

for $\Upsilon=\frac{\text { const }}{\sqrt{N}}$. Then we conclude the proof of (i) thanks to Claim B.

Now we shall prove (ii). Observe that there is a constant, depending only on $D(\mathfrak{P})$, such that

$$
\sup \left\{\operatorname{dist}_{\left(d s, \Xi_{N}^{j}\right)}\left(p_{0}, p\right): N \in \mathbb{N}, j \in\{1, \ldots, 2 N\}, p \in \Xi_{N}^{j}\right\} \leq \text { const },
$$

where $\Xi_{N}^{j}=\overline{D(\mathfrak{P})} \backslash \varpi_{j}^{*}$. Bearing in mind the above and using $\left(\mathrm{P} 2_{j}\right)$, we can obtain (ii). From (ii), it is not hard to deduce (iii).

Now, we deal with (iv). We will construct the multigon $\widetilde{\mathfrak{P}}$. Let

$$
\mathcal{S}=\left\{p \in D(\mathfrak{P}) \backslash \overline{D\left(\mathfrak{P}^{\varepsilon}\right)}: s<\operatorname{dist}_{\left(F_{2 N}, \overline{D(\mathfrak{P}))}\right.}\left(p, \partial\left(D\left(\mathfrak{P}^{\varepsilon}\right)\right)\right)<2 s\right\} .
$$

Note that $\mathcal{S}$ is a nonempty open subset of $D(\mathfrak{P}) \backslash \overline{D\left(\mathfrak{P}^{\varepsilon}\right)}$, satisfying $I(\mathcal{S})=\mathcal{S}$. As a consequence of (i), we deduce that $z(\mathcal{S})$ contains $k$ Jordan curves $\Gamma=\left\{\Gamma_{1}, \ldots, \Gamma_{k}\right\}$ satisfying that $f_{+}\left(\Gamma_{1}\right), \ldots, f_{+}\left(\Gamma_{k}\right)$ and $I\left(f_{+}\left(\Gamma_{1}\right)\right), \ldots, I\left(f_{+}\left(\Gamma_{k}\right)\right)$ bound a domain $\mathcal{D}$ such that $\overline{D\left(\mathfrak{P}^{\epsilon}\right)} \subset \mathcal{D} \subset \overline{\mathcal{D}} \subset D(\mathfrak{P})$. Then we can approximate $\Gamma$ by a multigon $\widetilde{\mathfrak{P}} \subset z(S)$ satisfying statements (iv.1) and (iv.2).

Finally, we prove assertion (iv.3), or equivalently $F_{2 N}(\partial(D(\widetilde{\mathfrak{P}}))) \subset B_{R}$. Take $p \in \partial(D(\widetilde{\mathfrak{P}}))$. If $p \in D(\mathfrak{P}) \backslash \bigcup_{j=1}^{2 N} \varpi_{j}^{*}$, we conclude that

$$
\left\|F_{2 N}(p)\right\| \leq\left\|F_{2 N}(p)-X(p)\right\|+\|X(p)\| \leq \frac{\text { const }}{N}+r \leq R .
$$

Suppose now $p \in \varpi_{j}^{*}, j \in\{1, \ldots, 2 N\}$. From (iv.2), it is possible to find a curve $\gamma:[0,1] \rightarrow D(\mathfrak{P})$ such that $\gamma(0) \in \partial\left(D\left(\mathfrak{P}^{\varepsilon}\right)\right), \gamma(1)=p$, and length $\left(\gamma, d s_{F_{2 N}}\right) \leq 2 s$. Let $\bar{p}=\gamma(\bar{t})$ be a point in the curve $\gamma$ where

$$
\bar{t}=\sup \left\{t \in[0,1]: \gamma(t) \in \partial\left(\varpi_{j}^{*}\right)\right\} .
$$

Using the former, we deduce that $\left\|F_{2 N}(\bar{p})-F_{2 N}(p)\right\| \leq 2 s$. Then, taking into account (ii), one has

$$
\left\|F_{j}(\bar{p})-F_{j}(p)\right\| \leq \frac{\text { const }}{N}+2 s .
$$


At this point, we are able to get bounds for coordinates of $F_{j}(p)$ in the frame $S_{j}$. Henceforth, we will use the frame $S_{j}$. We deal with the coordinates $\left(F_{j}(p)\right)_{3}$ and $\left(F_{j}(p)\right)_{*}=\left(\left(F_{j}(p)\right)_{1},\left(F_{j}(p)\right)_{2}\right)$. From $\left(\mathrm{P} 6.2_{j}\right)$ and (ii), we obtain a bound of the third coordinate of $F_{j}(p)$ :

$$
\left|\left(F_{j}(p)\right)_{3}\right|=\left|\left(F_{j-1}(p)\right)_{3}\right| \leq\left|\left(F_{j-1}(p)\right)_{3}-(X(p))_{3}\right|+\left|(X(p))_{3}\right| \leq \frac{\text { const }}{N}+r .
$$

On the other hand, using inequality (5), the fact that $\bar{p} \in D(\mathfrak{P}) \backslash \varpi_{j}^{*}$ and assertion (ii), one has

$$
\begin{aligned}
& \left\|\left(F_{j}(p)\right)_{*}\right\| \\
& \quad \leq\left\|\left(F_{j}(p)\right)_{*}-\left(F_{j}(\bar{p})\right)_{*}\right\|+\left\|\left(F_{j}(\bar{p})\right)_{*}-\left(F_{j-1}(\bar{p})\right)_{*}\right\|+\left\|\left(F_{j-1}(\bar{p})\right)_{*}\right\| \\
& \quad \leq \frac{c o n s t}{N}+2 s+\frac{c o n s t}{N^{2}}+\left\|\left(F_{j-1}(\bar{p})\right)_{*}\right\| .
\end{aligned}
$$

Observe that $\left\|\left(F_{j-1}(\bar{p})\right)_{*}\right\| \leq \frac{\text { const }}{\sqrt{N}}$. To check this, we follow the next arguments. If $\left\|F_{j-1}(\bar{p})\right\| \geq 1 / \sqrt{N}$, then $\left\|\left(F_{j-1}(\bar{p})\right)_{*}\right\| \leq \frac{\text { const }}{\sqrt{N}}\left(\frac{\text { const }}{N}+r\right) \leq \frac{\text { const }}{\sqrt{N}}$, as a consequence of $\left(\mathrm{P} 6.1_{j}\right)$ and (ii). If, instead, we have $\left\|F_{j-1}(\bar{p})\right\|<1 / \sqrt{N}$, then $\left\|\left(F_{j-1}(\bar{p})\right)_{*}\right\| \leq \frac{\text { const }}{\sqrt{N}}$ directly holds. In any case, following (6) and using the former, we can deduce that

$$
\left\|\left(F_{j}(p)\right)_{*}\right\| \leq \frac{\text { const }}{N}+2 s+\frac{\text { const }}{N^{2}}+\frac{\text { const }}{\sqrt{N}} \leq 2 s+\frac{\text { const }}{\sqrt{N}} .
$$

To finish, Pythagoras' theorem gives us

$$
\begin{aligned}
& \left\|F_{2 N}(p)\right\| \leq\left\|F_{2 N}(p)-F_{j}(p)\right\|+\left\|F_{j}(p)\right\| \\
& \quad \leq \frac{c o n s t}{N}+\sqrt{\left|\left(F_{j}(p)\right)_{3}\right|^{2}+\left\|\left(F_{j}(p)\right)_{*}\right\|^{2}}<\sqrt{r^{2}+(2 s)^{2}}+\varepsilon=R
\end{aligned}
$$

for an $N$ large enough.

It is easy to check that $Y=F_{2 N}$ satisfies all claims in Lemma 2, and then this ends the proof of the lemma.

\section{Proof of the Main theorem}

At this point, we prove our main result (Theorem 11).

Proof. Let $r_{1}>1$ and $\rho_{1}>0$, and define $r_{n}=\sqrt{r_{n-1}^{2}+(2 / n)^{2}}+1 / n^{2}$ and $\rho_{n}=$ $\rho_{1}+\sum_{i=2}^{n} 1 / i, n \geq 2$. Our purpose consists of using Lemma 2 to define a sequence

$$
\chi_{n}=\left(X_{n}: \overline{D\left(\mathfrak{P}_{n}\right)} \rightarrow \mathbb{R}^{3}, \mathfrak{P}_{n}, \varepsilon_{n}, \xi_{n}\right),
$$

where $X_{n}$ is a conformal minimal immersion, $\mathfrak{P}_{n}$ is a multigon, $\left\{\varepsilon_{n}\right\},\left\{\xi_{n}\right\}$ are decreasing sequences of nonvanishing terms satisfying $\varepsilon_{n}, \xi_{n}<1 / n^{2}$, and:

$\left(\mathrm{A}_{n}\right) \rho_{n}<\operatorname{dist}_{\left(X_{n}, \overline{\left.D\left(\mathfrak{P}_{n}^{\xi n}\right)\right)}\right.}\left(p_{0}, \partial\left(D\left(\mathfrak{P}_{n}^{\xi_{n}}\right)\right)\right)$,

$\left(\mathrm{B}_{n}\right) X_{n}\left(D\left(\mathfrak{P}_{n}\right)\right) \subset B_{r_{n}}$

$\left(\mathrm{C}_{n}\right) X_{n}(p)=\operatorname{Re}\left(\int_{p_{0}}^{p} \Phi^{n}\right)$, where $I^{*}\left(\Phi^{n}\right)=\bar{\Phi}^{n}$,

$\left(\mathrm{D}_{n}\right)\left\|X_{n}-X_{n-1}\right\|<\varepsilon_{n}$ in $D\left(\mathfrak{P}_{n-1}^{\xi_{n-1}}\right)$,

$\left(\mathrm{E}_{n}\right) \lambda_{X_{n}} \geq \alpha_{n} \lambda_{X_{n-1}}$ in $D\left(\mathfrak{P}_{n-1}^{\xi_{n-1}}\right)$, where $\left\{\alpha_{i}\right\}_{i \in \mathbb{N}}$ is a sequence of real numbers such that $0<\alpha_{i}<1$ and $\left\{\prod_{i=1}^{n} \alpha_{i}\right\}_{n}$ converges to $1 / 2$,

$\left(\mathrm{F}_{n}\right) \overline{D\left(\mathfrak{P}_{n-1}^{\xi_{n-1}}\right)} \subset D\left(\mathfrak{P}_{n}^{\xi_{n}}\right) \subset \overline{D\left(\mathfrak{P}_{n}\right)} \subset D\left(\mathfrak{P}_{n-1}\right)$. 
The first element of the sequence is taken as follows. If $\sigma=0$, we deal with $X_{1}: M_{0} \rightarrow \mathbb{R}^{3}$, the double oriented cover of Meeks' Möbius strip [6]. If $\sigma>0$, then we take $X_{1}: M_{\sigma} \rightarrow \mathbb{R}^{3}$ as the oriented double cover of the nonorientable surface of genus $\sigma+1$ described in [3. It is not hard to see that, in both cases, the closed Riemann surface can be written as

$$
\bar{M}=\left\{(z, w) \in \overline{\mathbb{C}}^{2}: w^{2}=\prod_{i=1}^{\sigma+1}\left(z-d_{i}\right)\left(z-\overline{d_{i}}\right)\right\}
$$

for suitable $d_{1}, \ldots, d_{\sigma+1}$, and the reversing orientation involution has the form $I(z, w)=(\bar{z},-\bar{w})$. The simply connected domain $\Delta$ is chosen in such a way that $\left\{d_{1}, \ldots, d_{\sigma+1}, \overline{d_{1}}, \ldots, \overline{d_{\sigma+1}}\right\} \subset \Delta$, and $\overline{z^{-1}(\Delta)} \subset M_{\sigma}$. The real numbers $\beta_{1}, \ldots, \beta_{k-1}$ are chosen in $\mathbb{C} \backslash \Delta$ and outside the $z$-projection of the ends of $X_{1}$. So, a convenient $\mathfrak{P}_{1}, r_{1}$ and $\rho_{1}$, joint with $X_{1}: \overline{D\left(\mathfrak{P}_{1}\right)} \rightarrow \mathbb{R}^{3}$ completes the construction of the first element in our sequence. We also choose $\xi_{1}<1$ small enough satisfying $\left(\mathrm{A}_{1}\right)$. The choice of $\varepsilon_{1}<1$ is irrelevant.

Lemma 2 allows us to construct the terms of the sequence in a recursive way. For more details see 2 .

Now, we define $D=\bigcup_{n=1}^{\infty} D\left(\mathfrak{P}_{n}^{\xi_{n}}\right)$. Observe that $D$ is the limit of an expansive sequence of domains of $\bar{M}$ with the same conformal type. Then, this conformal type remains at the limit, in other words, $D$ has the conformal type of a compact Riemann surface of genus $\sigma$ minus $k$ closed disks. Thanks to properties $\left(\mathrm{A}_{n}\right)-\left(\mathrm{F}_{n}\right)$, it is not difficult to check that $\left\{X_{n}\right\}$ converges to a complete bounded minimal immersion $X: D \rightarrow \mathbb{R}^{3}$ satisfying $X \circ I=X$. So, it induces a complete nonorientable bounded minimal immersion $\mathcal{X}: \mathcal{D} \rightarrow \mathbb{R}^{3}$. This concludes the proof.

\section{REFERENCES}

[1] H. M. Farkas, I. Kra, Riemann surfaces. Graduate Texts in Math., 72, Springer-Verlag, Berlin, 1980. MR0583745 (82c:30067)

[2] F.J. López, F. Martín, S. Morales, Adding handles to Nadirashvili's surfaces. J. Diff. Geom. 60 (2002), no. 3, 155-175. MR1924594 (2003f:53013)

[3] Francisco J. López, Francisco Martín, Complete nonorientable minimal surfaces and symmetries. Duke Math. J. 79 (1995), no. 3, 667-686. MR1355180 (98b:53008)

[4] F. Martín, S. Morales, A complete bounded minimal cylinder in $\mathbb{R}^{3}$. Michigan Math. J. 47 (2000), no. 3, 499-514. MR 1813541 (2001m:53015)

[5] N. Nadirashvili, Hadamard's and Calabi-Yau's conjectures on negatively curved and minimal surfaces. Invent. Math. 126 (1996), 457-465. MR1419004(98d:53014)

[6] W. H. Meeks, The classification of complete minimal surfaces in $\mathbb{R}^{3}$ with total curvature greater than $-8 \pi$. Duke Mathematical Journal 48 (1981), 523-535. MR0630583 (82k:53009)

[7] R. Osserman, A survey of minimal surfaces. Dover Publications, New York, second edition, 1986. MR0852409 (87j:53012)

[8] H. L. Royden, Function theory on compact Riemann surfaces. J. Analyse Math. 18 (1967), 295-327. MR0214757(35:5606)

Departamento de Geometría y Topología, Facultad de Ciencias, Universidad de Granada, 18071 Granada, Spain

E-mail address: fjlopez@ugr.es

Departamento de Geometría y Topología, Facultad de Ciencias, Universidad de Granada, 18071 Granada, Spain

E-mail address: fmartin@ugr.es

Departamento de Geometría y Topología, Facultad de Ciencias, Universidad de Granada, 18071 Granada, Spain

E-mail address: santimo@ugr.es 